\title{
El Currículo de las Matemáticas Escolares y el Gobierno del Sujeto Moderno
}

\section{School Mathematics Curriculum and the Governing of the Modern Subject}

\author{
Paola Valero* \\ Gloria García $^{* *}$
}

\begin{abstract}
Resumen
Pensar las matemáticas escolares con la noción foucauldiana de gobernamentalidad permite explorar el funcionamiento del currículo como tecnología de gobierno. Así, es posible entender cómo las prácticas y discursos de la educación matemática fabrican al sujeto racional, objetivo, universal que encarna al ciudadano cosmopolita Moderno. Con el estudio genealógico de las matemáticas escolares en el proyecto colonizador español en Colombia, y sus posteriores mutaciones en la consolidación del estado nación en el siglo 20, se muestra cómo el currículo de matemáticas efectúa la entrada de Colombia en el camino del desarrollo y cómo inscribe la norma del pensamiento y maneras de ser Modernas en los estudiantes colombianos. Este doble proceso mantiene la exclusión de subjetividades que no se alinean con el ser Moderno. El análisis cuestiona la visión ingenua del empoderamiento de todos que se le atribuyen a las matemáticas escolares.
\end{abstract}

Palabras-clave: Investigación Sociopolítica en Educación Matemática. Gobernamentalidad. Subjetividad. Tecnologías del Yo. Currículo Escolar de Matemáticas.

\begin{abstract}
Thinking school mathematics with the Foucauldian notion of governmentality allows the exploration of the functioning of the mathematics curriculum as a government technology. The practices and discourses of mathematics education fabricate the rational, objective, universal subject, who becomes the Modern cosmopolitan citizen. A genealogical analysis addresses the insertion of school mathematics in the Spanish colonial project in Colombia and in its subsequent mutations in the consolidation of the nation's state in the 20th century. The study shows how the Colombian mathematics curriculum affects the entry of the nation in the path towards development. It also points to the inscription of Modern norms and forms of thinking and being in Colombian children. This dual process maintains the exclusion of subjectivities that do not align with the Modern self. This analysis questions the naïve narrative of empowerment that is frequently attributed to school mathematics.
\end{abstract}

\footnotetext{
* Doctorado en Educación Matemática de la Danish University of Education. Profesora Titular y directora del programa de doctorado Ciencia y Tecnología y Educación para la salud, la organización y la ética en la Universidad de Aalborg, Dinamarca. Dirección postal: Nyhavnsgade 14, 9000, Aalborg, Dinamarca. E-mail: paola@learning.aau.dk.

Magister en Filosofía Latinoamericana de la Universidad Santo Tomás de Colombia. Profesora titular de la Universidad Pedagógica Nacional, Bogotá D.C., Colombia. Dirección postal: Cra 28, n. 45-A-26, Apt. 502, Bogotá D.C., Colombia. E-mail: glogaroliveros@hotmail.com.
} 
Keywords: Sociopolitical Research in Mathematics Education. Governmentality. Subjectivity. Technologies of the Self. School of Mathematics Curriculum.

\section{Introducción}

[L]a educación matemática debe responder a nuevas demandas globales y nacionales, como las relacionadas con una educación para todos, la atención a la diversidad y a la interculturalidad y la formación de ciudadanos y ciudadanas con las competencias necesarias para el ejercicio de sus derechos y deberes democráticos. (MEN, 2006, p. 46).

Para que ocorram as inserções dos cidadãos no mundo do trabalho, no mundo das relações sociais e no mundo da cultura e para que desenvolvam a crítica diante das questões sociais, é importante que a Matemática desempenhe, no currículo, equilibrada e indissociavelmente, seu papel na formação de capacidades intelectuais, na estruturação do pensamento, na agilização do raciocínio do aluno, na sua aplicação a problemas, situações da vida cotidiana e atividades do mundo do trabalho e no apoio à construção de conhecimentos em outras áreas curriculares. (BRASIL, 1998, p. 28).

Estas enunciaciones hacen parte de los documentos oficiales de los currículos de matemáticas en Colombia y Brasil. Para educadores matemáticos, tanto profesores como investigadores, estas frases se constituyen en derroteros a seguir para realizar las buenas intenciones y contribuciones que la enseñanza de las matemáticas tienen que sembrar en los niños de cada país. Estas enunciaciones expresan visiones deseadas sobre los distintos sujetos involucrados en la educación misma, y sobre el papel de la educación matemática en la construcción de un mundo mejor. Como lineamientos que deben ser implementados, las enunciaciones expresan, también, algunas de las verdades que circulan en las diversas prácticas de la enseñanza y aprendizaje de las matemáticas escolares: la educación matemática debe ser para todos; debe ser inclusiva de variedades culturales; debe contribuir a la ciudadanía; debe poder formar para el trabajo; y, por supuesto, formar la mente y la razón de quienes están involucrados.

Este tipo de enunciaciones dentro de documentos curriculares son apenas una parte de aquello que podríamos llamar el currículo de las matemáticas escolares. Una variedad de marcos conceptuales nos podrían dar un sentido de esa noción de currículo: Hay desde visiones que llamarían currículo a las listas de temas a enseñar en los distintos grados de la escolaridad, hasta visiones que abarcan el conjunto de justificaciones sociales, las organizaciones en la escuela y las actividades didácticas en el aula de matemáticas para llevar el conocimiento matemático de su ámbito disciplinar al espacio de las relaciones educativas (GÓMEZ, 2000). Una perspectiva teórica diferente, basada en los estudios de las historias culturales de Michel Foucault, permite desplegar estrategias analíticas para entender cómo la 
pedagogía transforma los conocimientos disciplinares de las matemáticas para darles entrada en la escuela (POPKEWITZ, 2004) y, así, generar tesis culturales sobre los sujetos deseados de la educación matemática. La fabricación de subjetividades es, entonces, uno de los efectos constructivos de poder de las tecnologías pedagógicas que gobiernan tanto a las poblaciones como al individuo.

En este artículo exploramos la forma como el currículo de matemáticas, como una forma de gobierno de la conducta de profesores y niños, constituye al sujeto racional, cosmopolita moderno. Si bien esta fabricación es un efecto productivo de poder, ella resulta también en la exclusión de otras formas de subjetividad. La gran narrativa de la inclusión y la promesa de un mejor futuro, que han acompañado a las prácticas educativas de las matemáticas a lo largo del siglo XX, operan clasificando y excluyendo los sujetos cuyas formas de vida no se insertan en la norma de la modernidad. Comenzamos por puntualizar algunas de las herramientas teóricas y estrategias analíticas que desplegamos en nuestro estudio. Luego presentamos algunos elementos de una incipiente historia del presente de las matemáticas escolares en Colombia. Tal exploración tiene el fin de mostrar cómo las matemáticas escolares, en la trama del proyecto colonizador español y la formación de un estado nación, inscribieron en niños y niñas las marcas del ser moderno, gobernando así a los sujetos escolares. Finalizamos con la discusión de cómo este análisis nos invita a pensar la in(ex)clusión en la educación matemática en países como Colombia.

\section{Estudio de lo político en educación matemática}

La investigación en educación matemática es un campo creciente, y dentro de este los estudios sociopolíticos cobran cada vez más importancia. Tales estudios se interesan por dar cuenta de cómo la educación matemática es una práctica política. Desde tal perspectiva han surgido dos intereses investigativos relacionados que vale la pena mencionar. En primer lugar está la investigación sobre la investigación (PAIS; VALERO, 2012; VALERO, 2008) cuyo interés es estudiar los discursos de la investigación y entender cómo ésta es una de las prácticas de conocimiento que genera tanto los lenguajes para nombrar objetos de estudio, como también las maneras de pensar que se consideran verdaderas sobre tales objetos. Podríamos ejemplificar este punto con estudios que se han preguntado cómo ciertas ideas que hoy en día tomamos como verdaderas de hecho han surgido en configuraciones epistemológicas de las ciencias educativas frente a lo que se define como problemáticas de la investigación en educación matemática. Por ejemplo, Knijnik et al. (2012) presentan un 
examen crítico de las verdades naturalizadas de la educación matemática como son es importante traer la realidad del alumno al aula para dar significado a los contenidos y generar interés por aprender, y así poder transformar socialmente al mundo (DUARTE, 2009); o aquella idea de que es importante usar materiales concretos para facilitar el aprendizaje y tener mejores resultados (KNIJNIK; WANDERER; DUARTE, 2008); o aquella verdad de que jla matemática está en todo lugar! Ellas muestran, a través de un análisis genealógico inspirado en distintas herramientas teóricas de Michel Foucault, que los enunciados que circulan en los discursos de investigación y práctica de la educación matemática se ensamblan en la trama de los conceptos de las ciencias educativas puestas en operación en la tarea de estudiar y mejorar el aprendizaje y enseñanza de las matemáticas, la misión explícita de la investigación en educación matemática.

En segundo lugar está el asunto del entendimiento del poder en la educación matemática a través de la fabricación de subjetividades. La mayoría de las lecturas que se caracterizan como políticas dentro de la educación matemática asumen que la dimensión política de tales prácticas tiene su esencia en el hecho de que el conocimiento matemático es poderoso y que empodera a quien lo posee (VALERO, 2008). El conocimiento se concibe como aquella posesión de la mente del individuo que le permite actuar de manera poderosa dentro de una sociedad formateada por las matemáticas (SKOVSMOSE, 1999). Además de visiones particulares de poder en tal posición, el alineamiento con una epistemología racionalista tradicional ha permitido sostener el mito de que el conocimiento verdadero está en la mente del individuo y es la fuente de acción correcta y verdadera. Gran parte de la educación matemática se ha fundamentado en epistemologías racionalistas, incluso algunas de las visiones sociopolíticas.

Radford (2008a, 2008b) ha criticado fuertemente el efecto de tales epistemologías racionalistas en las ontologías y epistemologías de las didácticas de las matemáticas. Él argumenta que la posición sociocultural implica sacar al conocimiento de la mente y localizarlo en las relaciones del yo con el otro en la cultura, con su simbolismo y materialidad. Radford (2008b, p. 225) ha planteado entender el aprendizaje como "el dotar de significado a los objetos conceptuales culturales" Para eso, el yo se abre al encuentro del otro y de la cultura para objectificar tales objetos. Al hacerlo el yo hace un movimiento reflexivo que se podría llamar subjetivación. Por esto el aprendizaje es tanto un proceso de conocer como de ser. Este desplazamiento del entendimiento del conocer del dominio de la mente al dominio del ser y la cultura permite comenzar a pensar en lo que es tanto el conocimiento matemático 
como el conocimiento matemático escolar como inseparables de las prácticas y sus sistemas de significación históricos. También invita a pensar en el conocimiento y sus efectos de poder en la modelación de subjetividades. Si el conocer y el conocimiento no son, en esencia, lo que emancipa al ser humano o lo que le otorga capacidad para actuar racionalmente, entonces ¿cuál es la relación entre conocer, conocimiento y poder?

Desde una perspectiva un poco diferente a la de Radford (2008a, 2008b), el conocimiento y el conocer se relacionan con el poder porque efectúan formas de ser; en otras palabras, forman subjetividades. El ser y el conocer son como las dos caras de la misma moneda. Las formas de conocimiento de la cultura no sólo llevan consigo el qué conocer y cómo hacerlo, sino que también imponen formas de ser en quienes conocen. Un ejemplo de esta relación lo presentan Daston y Gallison (2007) en su investigación sobre la conformación histórica de la objetividad como parte de las prácticas de las ciencias naturales en la segunda mitad del siglo XIX. Para ellos la objetividad, como se formó en ese tiempo, es una virtud epistémica que va mano a mano de la subjetividad. Una virtud epistémica puede entenderse como las normas que aquellas personas que participan de una práctica científica internalizan y hacen cumplir en ellos mismos y los demás. Las normas tienen una fundamentación en los valores éticos de la práctica que realizan y en la búsqueda de formas eficientes y prácticas de asegurar el conocimiento. Las virtudes epistémicas vinculan conocimiento con el ser pues moldean la subjetividad para acomodarla a las formas de producción de conocimiento (DASTON; GALISON, 2007). Así, hacer ciencia objetivamente requirió del surgimiento de nuevas formas de subjetividad, asociadas con técnicas particulares del ser del científico, que permitieran sobreponer la observación y privilegiar los objetos de conocimiento al suprimir la subjetividad del observador. De esta manera, conocer objetivamente significó un desplazamiento de la centralidad del ser y del deseo del observador/investigador por dar una interpretación, para pasar a conocer con el "deseo de no tener deseo". En otras palabras, el ser científico objetivo se constituyó dentro de prácticas científicas que requerían reprimir la subjetividad: "Como un proceso de eliminación algebraica, la negación de la subjetividad por el sujeto, se convirtió en objetividad" (DASTON; GALISON, 2007, p. 204).

Una lectura política que enraíza las prácticas de las matemáticas escolares dentro de las redes y tramas de la escolaridad propondría un entendimiento de poder con relación a la creación de subjetividades. Pensando esta idea con algunas de las herramientas teóricas de Michel Foucault sería posible afirmar que el problema del poder de la educación matemática es el problema de cómo y con qué tecnologías de gobierno de la población y del yo, las 
matemáticas escolares, como parte del currículo escolar, generan los tipos de sujetos históricos y culturales de un tiempo determinado. Aunque ha habido algunos estudios sociopolíticos que han tomado las herramientas teóricas de Michel Foucault en educación matemática (HARDY, 2004; WALSHAW, 2004), todavía hay mucho terreno para explorar en el entendimiento de cómo en las prácticas escolares de las matemáticas han sido un espacio importante de formación de tesis culturales sobre el niño moderno deseado que la educación masiva del siglo $\mathrm{XX}$ ha tenido como función construir. Este último punto quisiéramos desarrollar con más detalle, ejemplificando algunas de las configuraciones históricas que se han generado en un país como Colombia, alrededor de la construcción del sujeto matemáticamente competente.

\section{Estrategia analítica}

Enfoquémonos ahora en la pregunta de cómo las prácticas - sociales, culturales, políticas, históricas y económicas - de las matemáticas escolares contribuyen para conformar ciertos tipos de subjetividades en los niños aprendices de matemáticas. Para esto recurriremos a bosquejar elementos de un trabajo genealógico de la educación matemática que está casi por hacer, es decir, que es todavía un terreno cuasi virgen e inexplorado en la investigación actual en educación matemática.

\subsection{Estrategia genealógica para historizar el presente}

Una estrategia de trabajo genealógica inspirada en Foucault se caracteriza por al menos tres elementos. En primer lugar, la historización busca romper con la idea tradicional de la historiografía de la linealidad causal de los sucesos para encontrar los orígenes verdaderos de los hechos. El intento de historizar no busca narrar un pasado sino presentar una historia del presente. Como dice Saar (2002, p. 234):

[la genealogía] es una escritura específica de la historia de ciertos objetos. Esta historiografía da cuenta de 'nuestra' historia, es decir, de los procesos de constitución y construcción de la moralidad, mentalidad o 'alma' presentes, en todas sus discontinuidades, transformaciones funcionales y contingencias.

La estrategia de historización nos llevó a revisar los trabajos realizados en Colombia sobre la historia de las matemáticas, las ciencias y la educación (SÁNCHEZ; ALBIS, 2012) y hacer una lectura de ellos que establece conexión con las distintas tesis culturales y formas epistemológicas de cada tiempo. Este trabajo historizador de tipo rizomático - en el sentido 
de Deleuze y Guattari (1987) - permite explorar las tramas discursivas en las que se constituyen los significados y prácticas de la educación matemática. Este tipo de estrategia historizadora la ponemos en las secciones Colonia, ciencia y matemáticas y Modernización, estado y matemáticas escolares.

En segundo lugar, la crítica tiene la intención de desnaturalizar las verdades que circulan en enunciados discursos de nuestras prácticas y que constituyen las racionalidades dentro de las cuales aprendemos a ser sujetos de un tiempo. Saar (2002, p. 236-237) expresa el imperativo crítico de la genealogía de la siguiente manera: "Cuéntame la historia de la génesis y desarrollo de mi entendimiento de mí mismo [...] de tal manera que al oírte hablar, no quiera ser más como creía que tengo que ser, y que, al oírte hablar, me dé cuenta de que ésta [forma de ser] no es "necesaria"'. En otras palabras, la crítica evidencia que las verdades que tomamos por dadas sobre nuestras formas de vida son contingentes y no necesarias. De tal manera puede abrirse una oportunidad para pensar - y ser - aquello que dentro de los marcos establecidos no es aún posible.

La estrategia crítica asociada a la genealogía se pone en operación en todo el artículo, pues la investigación de cómo el currículo de matemáticas tiene efectos de poder en la construcción de la subjetividad moderna constituye en sí un movimiento crítico que problematiza la verdad generalizada de que las matemáticas escolares son poderosas dado el poder intrínseco del conocimiento escolar. La historización del presente de las tesis culturales del currículo de matemáticas sobre quienes son los niños deseados que fabrica el currículo constituye un desplazamiento problematizador del foco de estudio de la investigación en educación matemática. Al alejarnos de los estudios del currículo en términos de aprendizaje y enseñanza y enraizar las prácticas educativas de las matemáticas dentro del campo de la política cultural de la escuela, estamos operando un movimiento crítico que permite ver otras opciones para el entendimiento de dichas prácticas. Este estudio, por lo tanto, no es un tipo de estudio de educación matemática crítica en el sentido que tanto Skovsmose (1999) o Skovsmose y Valero (2012) han delineado anteriormente. Es un estudio crítico que apunta a una dirección de crítica post-estructural a la constitución de las prácticas educativas de las matemáticas.

Finalmente, la forma misma de la escritura de una genealogía se aleja de lo que se consideraría un estilo académico clásico para adoptar formas de representación impactantes y con efecto en el lector. Como dice Saar (2002, p. 238): enunciados y serie de proposiciones; el 'efecto de verdad' que estos textos tratan de 
lograr solo se materializa en la fusión de ciertas hipótesis históricas y un modo de representación drástico y dramatizante.

La estrategia de escritura también puede verse en todo el artículo, en particular en nuestro esfuerzo por construir un texto argumentativo que provoca al lector y lo confronta con formas de investigación más filosóficas y menos empiricistas que son frecuentes en las ciencias educativas y en la educación matemática en particular.

Con estos elementos en mente pasemos a abordar la pregunta que nos concierne que es cómo pensar en la manera como las matemáticas escolares construyen tesis culturales sobre los sujetos deseados.

\section{Educación matemática y subjetividad}

Bishop (2005) formuló una tesis interesante: las matemáticas occidentales han funcionado en muchos sistemas coloniales como una de las armas secretas del imperialismo cultural. Ante la verdad que circulaba hasta la década de 1980 - la cual todavía navega fuertemente tanto entre el público como entre muchas de las personas que trabajan con matemáticas - de que las matemáticas son un tipo de conocimiento universal y descontextualizado y, por lo tanto, desprovisto de un arraigo cultural o de una posibilidad de influir en la cultura, Bishop argumentó que las matemáticas occidentales habían tenido gran impacto en la colonización del mundo y en la expansión y dominancia de la cultura occidental frente a muchas culturas colonizadas. Bishop señaló tres procesos importantes:

- $\quad$ el comercio, que facilitó la imposición de sistemas de medida estandarizados frente a muchos sistemas de medición locales;

- la administración y gobierno de la población con sus sistemas de manejo de grandes cantidades de información que se hizo paulatinamente más numérica, junto con la imposición de las lenguas europeas y sus categorías de clasificación;

- $\quad$ y la educación de las poblaciones colonizadas, en particular la enseñanza y aprendizaje de las matemáticas.

Con respecto a la educación, los sistemas de clasificación y estratificación de la población para asegurar la élite fueron copiados a las colonias. En la educación matemática el uso de materiales producidos en las colonias, por ejemplo, permitió generar situaciones donde el suceso escolar claramente se relacionaba con conocer y manejar parte de la cultura dominante. Innumerables ejemplos se encuentran de la utilización de libros de texto plagados 
con problemas que piden a los niños de las colonias resolver situaciones presentadas en un contexto urbano de una de las grandes capitales europeas. En tales casos, los libros de texto no sólo se veían como los portadores de la cultura matemática de occidente, sino, también, como los propagadores de la cultura del poder colonial a la cual se deseaba enculturar a los colonizados.

Si bien la investigación en educación matemática, como aquella liderada por Alan Bishop o aquella que hace parte de la etnomatemática, ha tratado de deconstruir el mito de la neutralidad cultural de las matemáticas y las matemáticas escolares, los análisis de cómo y por qué procesos y mecanismos como los tres mencionados por Bishop (2005) han sido armas efectivas de imperialismo cultural no han sido tan contundentes. La propuesta de estudiar cómo tales mecanismos constituyen la subjetividad se vuelve entonces una posibilidad para ir más allá en tal comprensión. Tomemos el caso de Colombia y tratemos de delinear algunos elementos de tal tipo de análisis.

\subsection{Colonia, ciencia y matemáticas ${ }^{1}$}

Sánchez y Albis (2012), historiadores colombianos de las matemáticas, cuentan que la primera cátedra de matemáticas en el Nuevo Reino de Granada fue abierta por José Celestino Mutis, sacerdote jesuita, médico personal del virrey Mecía de la Cerda y líder de la gran empresa naturalista de la corona española, la Real Expedición Botánica. En el Colegio Mayor de Nuestra Señora del Rosario, en Santafé de Bogotá, Mutis dictó la primera cátedra de matemáticas, en 1761. A pesar del poco material escrito sobre la cátedra, los historiadores han podido rescatar dos aspectos importantes del proyecto de Mutis con su cátedra de matemáticas. Por un lado, Mutis privilegiaba las matemáticas como un método de pensamiento y como un conocimiento de gran utilidad "para todo tipo de personas: rústicos, ciudadanos, plebeyos, cortesanos, militares, artífices. Sabios, seculares, eclesiásticos, todos en una palabra de cualquier condición y estado" (MUTIS y FONNEGRA (1983) apud SÁNCHEZ; ALBIS, 2012, p. 110). Por otro lado, la enseñanza de matemáticas, desde ese momento, se alineó con la introducción de las ciencias en el contexto de una incipiente educación que enfatizaba la doctrina cristiana para mantener la sumisión de los nativos (Figura 1).

\footnotetext{
${ }^{1}$ Esta y la siguiente secciones del texto expanden las ideas presentadas en Valero et al. (2012).
} 


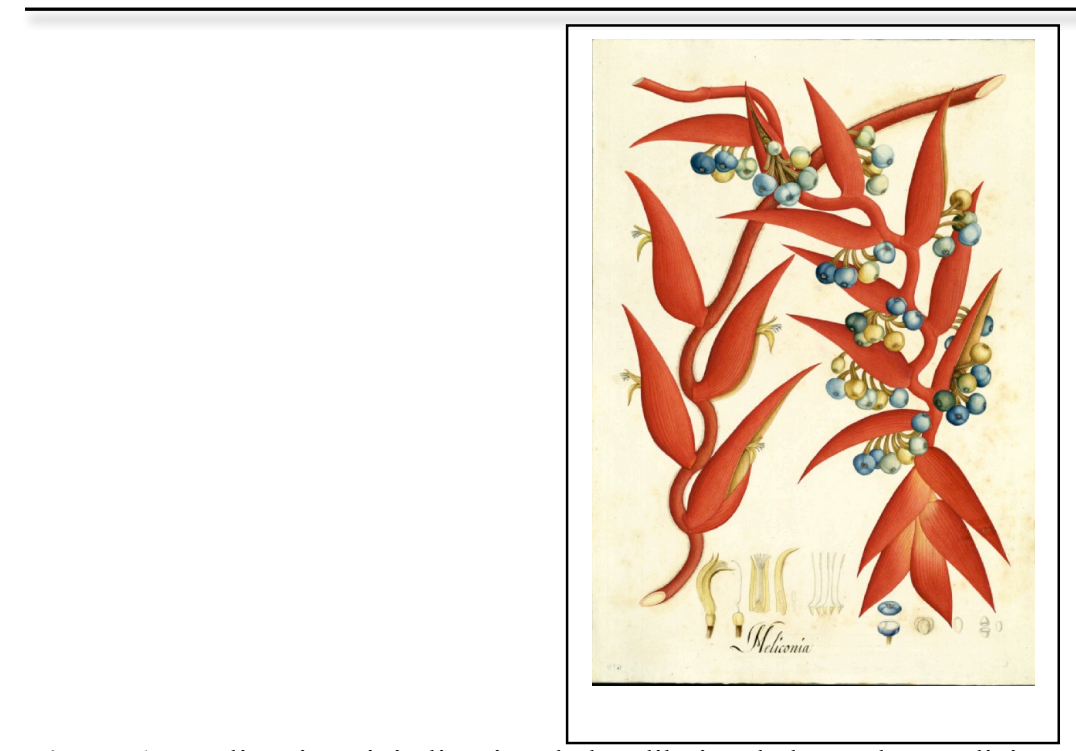

Figura 1 - Heliconia. Digitalización de los dibujos de la Real Expedición Botánica del Nuevo Reino de Granada (1783-1816).

Fuente: http: www.rjb.csic.es/icones/mutis

La narrativa de los historiadores de las matemáticas Sánchez y Albis (2012) nos pone en la trama del tejido epistemológico que va a mantenerse como una continuidad de la configuración de la educación matemática desde la colonia hasta nuestros tiempos. La narrativa también deja a un lado otras dimensiones que, particularmente en el siglo $\mathrm{XX}$ cobran importancia. Como Bishop propuso en su artículo provocador, las matemáticas escolares son parte del proyecto colonizador, en el caso que nos ocupa, del poder español en las Américas. A diferencia de los proyectos colonizadores de Inglaterra en África e India o de otros poderes coloniales en el resto del mundo, la intersección entre el proyecto católico evangelizador y el proyecto del avance de las ciencias empíricas es el terreno donde se construyen epistemologías sociales particulares sobre el ser educado matemáticamente.

Muchas historias de las ciencias y las matemáticas en las Américas privilegian una narrativa de la contribución del Nuevo Mundo a la empresa de conocimiento científico colectiva que se comenzó a configurar en ese tiempo. No obstante, el efecto de poder de tal contribución sobre el ser colonial a veces es ignorado. La Real Expedición Botánica (Figura 1) fue una de las grandes empresas científicas de las Américas, que pretendía ser parte del movimiento naturalista de la época - entre los cuales el sueco Carlos Linneo fue el mayor exponente - y así mostrar, minuciosamente, las maravillas botánicas del Nuevo Mundo. También fue una empresa colectiva de documentación de la geografía, la astronomía y la metereología de ese territorio (SÁNCHEZ; ALBIS, 2012). El naturalismo como práctica científica propuso un sistema universal de clasificación del mundo natural y todos sus seres. Nieto (2000) señala que el interés de los expedicionarios era enviar la información a Europa 
para cotejarla con la taxonomía ya establecida. El proceso se adelantó sin reconocimiento alguno de quienes habían sido los portadores originales del conocimiento sobre la flora y fauna de la región. Para tales personas y sus saberes culturales tradicionales se mantenían los calificativos de irracionales, salvajes y supersticiosos. Así, con la ayuda - no reconocida - de los nativos y con las técnicas de recolección y dibujo de la época se clasificaron y catalogaron tantas especies como fue posible. La virtud epistémica (DASTON; GALISON, 2007) que guiaba aquella práctica era la de develar el arquetipo ideal y perfecto que, hecho bajo la imagen y semejanza de Dios, se podía casi deducir a partir de la generalización de los casos particulares e imperfectos que el ser humano puede observar con sus ojos. Para el científico y los artistas que participaban en esta práctica, la documentación científica objetiva consistía en plasmar la perfección divina de cada especie (Figura 2).

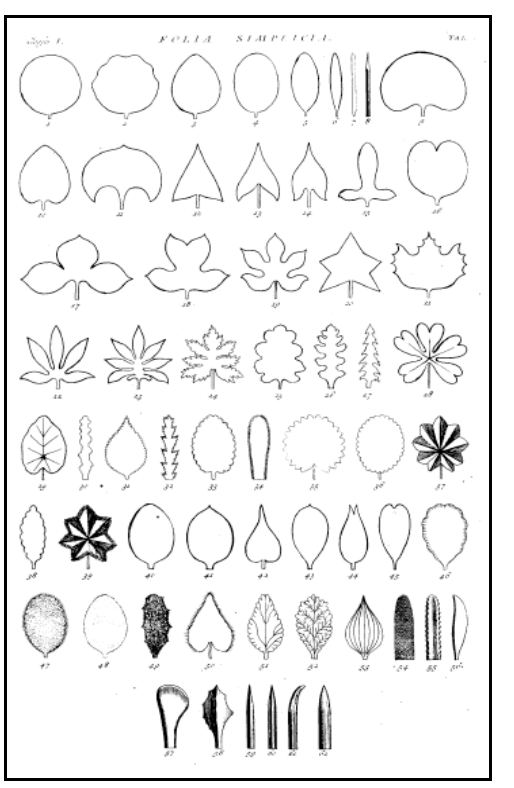

Figura 2 - Carl Linneaus, Hortus Cliffortianus, Folia Simplicia.

Fuente: http://www.botanicus.org/bibliography/b11957918

El interés de José Celestino Mutis por introducir las matemáticas en el territorio de la Nueva Granada se inserta dentro de esta lógica científica y contribuye a la generación de ciertas virtudes epistémicas y formas de entender la subjetividad de lo que se podría configurar como científico en las poblaciones del Nuevo Mundo. En primer lugar, hay que señalar que el naturalismo y su creación de taxonomías es una expresión científica de aquello que Foucault (1971) llamó la episteme clásica de la modernidad. La generación de taxonomías impone un orden estructural al mundo físico, y se basa en supuestos sobre el conocimiento que privilegian un orden lógico y abstracto como el principio de certidumbre para la organización del mundo, frente a la observación empírica de la variedad del mundo natural (SLOAN, 1976). Tal virtud epistémica es posible en la confluencia de dos tipos de 
razonamiento como el fundamento de una visión de ciencia: las matemáticas y sus formas de abstracción operan dentro de una visión teológica del mundo. Desde los griegos, y pasando por el Renacimiento y el inicio de las visiones antropocéntricas copernicanas, el mundo está estructurado con perfección divina y organizado bajo principios matemáticos. Por eso, el acceso del hombre al conocimiento verdadero se logra a través de las matemáticas. Como sugiere Harrison (2009), la búsqueda a principios de la modernidad de un lenguaje natural transparente y no ambiguo se basaba no sólo en una matematización de la naturaleza que asumía que los órdenes matemáticos representaban la Sabiduría Divina, sino también en que hay un orden en la naturaleza que está regida por los principio del orden matemático: "Si Dios es el Creador, se asumió que detrás de la notable diversidad de las cosas con vida debe haber algún orden comparable de alguna manera con el orden matemático que gobierna el movimiento de los cuerpos físicos". (HARRISON, 2009, p. 883).

La afirmación de Mutis (1983 apud SÁNCHEZ; ALBIS, 2012, p. 110) de que las matemáticas fueran un estudio "para todo tipo de personas" podría entenderse en este tejido epistemológico. La introducción de la cátedra de matemáticas en el Nuevo Reino de Granada marca la inserción del Nuevo Mundo dentro de la episteme clásica moderna, en unas condiciones donde el ser científico va de la mano con un ser religioso. La labor de educación de los nuevos súbditos coloniales con las matemáticas se realiza en el marco de la hibridación de ciencia y religión. Y esta es una característica de la construcción de la educación en países como Colombia que guarda continuidades históricas, al mismo tiempo que nuevas configuraciones, y por lo tanto discontinuidades, en su configuración en los discursos y prácticas educativas hasta nuestros días.

\subsection{Modernización, estado y matemáticas escolares}

Sánchez y Albis (2012) también cuentan que al finalizar la colonia, la enseñanza de las matemáticas en Colombia en las universidades estuvo asociada con la educación de las figuras prominentes de la creciente élite criolla - descendiente de los ancestros españoles quienes se esforzaron en mantener al país en marcha con el pensamiento y avances de Europa y, posteriormente, del naciente poder Norte Americano. Desde la segunda mitad del siglo XIX hasta el comienzo del XX, la visión de la construcción de un estado nación que promoviera el desarrollo económico a través del avance de la ingeniería para domar la naturaleza tropical agreste se asoció con la ubicación y crecimiento de las matemáticas en la Escuela Militar y, 
más adelante, en la naciente Universidad Nacional de Colombia. En este contexto, las discusiones sobre el papel de las matemáticas para el desarrollo del país se pueden interpretar como aquel elemento que podría propiciar el pensamiento racional, científico y progresista en la población.

Tal trama de argumentos y racionalidades, de hecho, no es nada único para Colombia. Durante la segunda industrialización y a medida que avanzaba el siglo XIX se comenzó a enunciar claramente el deseo de que un grupo creciente de personas en cada sociedad se dedicara al estudio de las nuevas ciencias y las matemáticas. En las universidades europeas, el fortalecimiento del espíritu científico y el avance de las ciencias experimentales frente a una organización de los campos de conocimiento que privilegiaban la filosofía (RÜEGG, 2004), se refleja en formulaciones que conectan la enseñanza de las matemáticas como una necesidad para el progreso científico. En el primer número de L'Enseignement Mathématique, la primera revista especializada de educación matemática, los editores C. A. Laisant y H. Fehr argumentaban que:

El futuro de la civilización depende en gran medida de la dirección del pensamiento que las nuevas generaciones recibirán con respecto a la ciencia. Dentro de la educación científica, el elemento matemático ocupa un papel dominante. Desde el punto de las ciencias puras o de sus aplicaciones, el siglo XX que está a punto de comenzar colocará demandas que nadie debe o puede evitar (LAISANT; FEHR, 1899, p. 5).

Más aun, la expansión del estudio de las matemáticas de las universidades a las escuelas también representa un movimiento hacia la inserción de tantas personas como fuera posible dentro de las racionalidades de la modernidad. La enseñanza y aprendizaje de las matemáticas, como parte del currículo escolar en la organización naciente de educación masiva, es una invención de finales del siglo XIX y comienzos del XX. Por ejemplo, Howson (1974) discute cómo en el caso de Inglaterra, los finales del siglo XIX fueron un tiempo en que personas interesadas en las matemáticas lograron hacer que la aritmética y otros temas matemáticos de utilidad práctica y mercantil entraran a ser parte de la nueva configuración curricular moderna. De manera similar, en muchos países del mundo, a comienzos del siglo $\mathrm{XX}$, se inició una organización curricular que llevara el desarrollo de habilidades matemáticas básicas como parte de aquellas cualificaciones fundamentales de una nueva mano de obra educada masivamente (RADFORD, 2004).

Popkewitz (2008, p. 1) ha estudiado los efectos de la educación y las ciencias educativas en la constitución del ciudadano cosmopolita moderno en los Estados Unidos en el cambio al siglo XX. El cosmopolitanismo se refiere a aquella "esperanza del Iluminismo por 
un ciudadano mundial cuyos compromisos trascienden las preocupaciones provinciales y locales con la adopción de valores ideales sobre la humanidad". En su análisis, Popkewitz articula nociones que, con base en las formas analíticas Foucaultianas, permiten examinar la fabricación de tesis culturales y sistemas de razón sobre los sujetos de la educación (POPKEWITZ, 2004).

Poner en operación estas estrategias analíticas permite examinar el currículo de matemáticas de una manera completamente diferente. El poder no es un simple acto de imposición de una voluntad sobre la otra, ni tampoco es sólo un acto de opresión. Foucault propone pensar el poder en términos de cómo diferentes formas de gobierno con sus mentalidades asociadas - la goberna-mentalidad — hacen posible conducir la conducta de la población, de otros y de uno mismo para formar las subjetividades que histórica y culturalmente se van haciendo posibles (FOUCAULT, 1982, 1997). Las tecnologías del yo son aquel conjunto de técnicas de gobierno que operan sobre la conducta de uno mismo que permiten a los individuos:

[...] efectuar por sus propios medios o con ayuda de otros, un cierto número de operaciones sobre sus propios cuerpos y almas, pensamientos y conductas, y una manera de ser, de tal manera que se transformen a ellos mismos para lograr cierto estado de felicidad, pureza, perfección o inmortalidad (FOUCAULT, 1997, p. 225).

Mientras que el discurso oficial y sus justificaciones para las matemáticas escolares enfatizan la necesidad de que los niños se apropien de un conocimiento valorado y útil para la vida personal, social, económica y cultural, pensar con las herramientas conceptuales de Foucault el currículo de matemáticas permite desnaturalizar este tipo de enunciados. Lo que se vuelve evidente es el efecto de poder del currículo de matemáticas en hacer al niño el ser racional, cosmopolita moderno. Es decir, el currículo permite operar sobre los cuerpos, almas, pensamientos y conductas del niño, el ser moderno que es deseado como el tipo de ser funcional y apropiado para el tipo de sociedad moderna. Sólo este ser es quien podrá alcanzar la promesa de un futuro mejor, con progreso económico y bienestar social - y también personal - que es la imagen pastoral de sociedad a alcanzar.

El currículo de matemáticas encarna y pone a disposición de todos quienes lo operan las formas cosmopolitas de razonamiento, que se basan en la creencia de que la razón humana, basada en la ciencia, tiene una capacidad universal emancipatoria para controlar y cambiar al mundo y a la sociedad. La posibilidad de acción del ser humano, la esperanza del progreso, la ciencia como el medio para dirigir y lograr progreso, y la planeación del tiempo son elementos que conforman una tesis sobre quién es el sujeto humano: un ser con una 
"mente sin hogar" - en inglés, "the homeless mind" (POPKEWITZ, 2008, p. 30). La "mente sin hogar" es un tipo de "individualidad que es tanto un objeto como una reflexión" y que ubica a los "individuos en una relación con categorías trascendentales que parecen no tener ninguna localidad histórica particular ni autor donde establecer un hogar". Esta tesis sobre los sujetos deseados se posibilita, entre otras, cuando la cuantificación desplaza formas de conocer cualitativas por formas de conocer cuantitativas que pueden entonces constituirse y, de ahí en adelante, modelarse como hechos (POOVEY, 1998). También, cuando la ciencias tanto sociales, humanas, como naturales y abstractas - hacen del mundo de las cosas y de los humanos el objeto de reflexión, estudio y planeación. Las matemáticas escolares como área del currículo escolar en aquella época, e incluso, todavía hoy en día, se convirtió en una de las áreas de la escolaridad que mejor podría fabricar tal tipo de subjetividad.

En la historia de Colombia, el ideal del sujeto cosmopolita europeo y norteamericano se reinscribe de maneras particulares. Es importante anotar que en los Estados Unidos la agenda de la reforma educativa, desde la primera década del siglo XX, se vinculó a la narrativa luterana de redención de una masa creciente de población urbana problemática. En ese, entonces, las nuevas poblaciones inmigrantes pobres de Europa - italianos, irlandeses, suecos, finlandeses etc. - era una masa urbana percibida como carente de todo tipo de buenos hábitos. La educación y las ciencias educativas debían rescatarlos (POPKEWITZ, 2008). En Colombia, como habíamos señalado anteriormente, educación, ciencia/matemáticas y religión católica configuran una trama en la que se teje una narrativa de salvación particular para el currículo de matemáticas. Desde la colonia, la educación de los nativos del Nuevo Mundo se encomendó a la Iglesia Católica, más precisamente se confió a la Compañía de Jesús (AHERN, 1991). La alianza política entre el poder colonial y la Iglesia Católica fue una estrategia doblemente efectiva para no sólo subordinar a los colonizados a un nuevo poder de gobierno, sino también - y ante todo - europeizarlos al crear un doble sistema de lealtades a Dios y a la Corona Española.

Un elemento importante en la trama de la constitución del sujeto deseado se hace evidente: el ser (colonizado) Colombiano, e incluso Latinoamericano, es aquel que ha aprendido "cómo ser un sujeto que siempre está pensando en ser lo que no es" (RIVAS, 2005, p. 117). Guillén (1996) en su ambicioso estudio de la formación del poder político en Colombia muestra cómo la asimilación al europeo fue una de las estrategias de gobierno que se instauró desde la colonia y que se ha reconfigurado durante la historia del país. A comienzos del siglo XX el parecerse a el otro - el blanco europeo (o norteamericano) - fue 
una de las ideas que guiaron los intentos de modernización de las élites nacionales. De manera similar, Zemelman y Quintar (RIVAS, 2005) señalan que la asimilación es una de las formas de ser y relacionarse que permitieron generar toda una cultura de subordinación y de negación de lo que se es.

Esta característica se conecta con otra muy importante y es la de, una vez asimilado, toda diferencia con el ideal de sujeto se niega, se silencia, e incluso se elimina. Guerra (1997) sugiere que las nociones de democracia y ciudadanía que surgieron en muchos países de América Latina durante la consolidación de estados nación, en el siglo XX, se enraízan en la idea de similitud y correspondencia. La unificación - a expensas de la diversidad - fue una estrategia importante de cohesión en sociedades con múltiples diversidades étnicas, culturales, sociales y económicas. Más aún, la religión Católica fue uno de los elementos de narrativas de unificación nacional, pues era ésta la creencia que permitía vincular a todos para alcanzar las promesas de un futuro mejor (DÍAZ, 2010). Dentro de esta narrativa de unificación, la educación, principalmente católica, se convirtió en un elemento importante para mantener y promover una modernización en el siglo XX.

La pregunta que surge es cómo el currículo de matemáticas como forma de gobierno de la población y del yo generó tesis sobre un ser, por un lado cosmopolita, racional, científico y matemático, y por otro lado, religioso y abierto a la promesa pastoral de la Iglesia Católica. Esta pregunta, pertinente en la mayoría de las sociedades Latinoamericanas, no ha estado presente en casi ninguno de los análisis sobre las matemáticas escolares en nuestro territorio $y$, sin embargo, es una pregunta clave hacia una posible interpretación del presente educativo de nuestras sociedades.

Vale la pena detenerse aquí y tratar de esculcar en la trama que posibilitó tal tipo de fabricación de sujeto. Saldarriaga-Velez (2010) presenta un estudio genealógico que aborda el problema de cómo fue posible asegurar el acceso de la gente común a un conocimiento verdadero que se conformaba entre las verdades universales y válidas de la ciencia y las verdades morales de la religión. El proyecto intelectual católico de finales del siglo XIX, representado, entre otras, por la restauración de la filosofía de Santo Tomás de Aquino - el neotomismo - integró los avances y propuestas del empiricismo científico del siglo XVIII y XIX, con una filosofía que pretendía retomar el poder de la racionalidad tradicional espiritual en un entorno crecientemente laico y liberal. La educación fue, sin duda, uno de los espacios donde tal filosofía podía tocar al hombre común. Dado el pacto político de 1886 entre el estado Colombiano y la Santa Sede, a través de un concordato que, entre otras, aseguraba que 
la educación pública estaría en manos del clero, la herencia colonial aseguró continuidad en el siglo XX. Tal acuerdo fue el terreno propicio para el despliegue de la filosofía neotomista en las prácticas educativas de la época. La síntesis entre acceso al conocimiento de Dios y entendimiento de las verdades perennes de la ciencia y las matemáticas, con sus métodos de conocimiento seguro y verdadero, posiciona a los saberes de los números, sus estructuras y la geometría como vías necesarias para la realización intelectual y espiritual. En sus escritos sobre el modernismo, monseñor Rafael María, uno de los educadores neotomistas más prominentes del cambio de siglo, a cargo del ministerio de instrucción pública a finales del siglo XIX en Colombia, decía:

La verdad metafísica es inmutable, y no relativa, sino absoluta. [...] El triángulo
siempre ha sido, aún antes de la creación, figura cerrada de tres líneas; el hombre,
animal racional; la envidia, pesar del bien ajeno. Por eso los principios de las
matemáticas se apellidan verdades necesarias. Se replica que las criaturas son
contingentes, temporales, mutables. Es verdad, pero lo mutable, lo temporal, lo
contingente, es la existencia, no la naturaleza [...] Ninguna verdad es mudable ni
relativa, pero hay algunas que son capaces de crecer en número en el entendimiento
humano. Esas han sido dejadas por Dios al cuidado de los hombres, [...] son los
descubrimientos científicos (CARRASQUILLA, 1916 apud SALDARRIAGA-
VÉLEZ, 2010, p. 85).

En el transcurso del siglo XX y el avance de la psicología como la ciencia de la mente, un elemento importante se añade a esta trama: todo ser humano común puede acceder a la verdad Divina a través de las ciencias y las matemáticas, pues la capacidad básica de cognición del ser humano es la dotación que se ha recibido del Todopoderoso para acceder al mundo (SALDARRIAGA-VÉLEZ, 2010). Así, a pesar del desplazamiento de la filosofía y la moral durante el siglo $\mathrm{XX}$, por las nuevas ciencias psicológicas y cognitivas se siguió asegurando la posibilidad de mantener estrechamente vinculados al aprendizaje de las ciencias y las matemáticas con la religión a través de una psicología cognitiva y su inserción en distintas pedagogías modernas.

A mediados del siglo XX, otros hilos entran en la trama de la educación matemática escolar y su fabricación del sujeto moderno en Colombia. A partir de la década de 1960, el proceso de consolidación de estado nación se conecta con el avance de las agendas de instituciones como el Banco Mundial para cooperación internacional por el desarrollo y la modernización. A partir de 1960, el gobierno nacional comenzó a responder a los retos generados por una creciente deserción escolar que se presentó con una expansión de la cobertura de la educación para abarcar las nuevas masas de población urbana dado el fuerte proceso de urbanización y abandono del campo generado por la violencia política rural. Las respuestas para expandir y también fortalecer la educación se alinearon con las políticas de 
agencias internacionales que impusieron la adopción e implementación de estrategias de planeación tecnocráticas en la educación. Una buena educación para el desarrollo no sólo conllevó el aumento en el manejo político y administrativo de la educación para satisfacer las necesidades del desarrollo social y económico del país, sino también, y más concretamente, la introducción de tecnologías educativas modernas que aseguraran hacer los procesos educativos más efectivos, flexibles y constantemente educativos (GARCÍA, 2003). Tales formulaciones se hicieron más claras, unos años más tarde, cuando la efectividad educativa, medida tanto cualitativa como cuantitativamente, también se conectó con la optimización de la inversión pública en la educación. De esta manera, la lógica de la educación para el desarrollo como parte del proceso de manejo del estado se acompañó de tecnologías concretas para gobernar la conducta de los niños escolares.

Estas dos tendencias fueron parte de la trama que posibilitó, unos años más tarde, la Renovación Curricular de la década de 1980. El gran primer currículo de matemáticas nacional organizado bajo los principios de la epistemología genética piagetiana y la visión de sistemas matemáticos emergió bajo el liderazgo intelectual de Carlos Vasco, filósofo y matemático jesuita, quien funcionó como consultor del Ministerio de Educación Nacional entre 1978 y 1993 (MOLANO, 2011). El currículo de matemáticas propuesto en esta renovación puede verse como una forma de gobierno con tecnologías que operan tanto a nivel de la población, como del yo. Por un lado, el currículo de matemáticas de la Renovación se desplegó como una organización detallada de los contenidos y las formas de enseñanza a seguir para lograr los objetivos de conocimiento establecidos. Materiales ministeriales, libros de texto, y esfuerzos de formación inicial y continuada de maestros trataron de asegurar que todo maestro de matemáticas de Colombia se alineara a la propuesta. La consolidación de las pruebas de estado al terminar el ciclo de secundaria se aseguraba de verificar el efecto de la visión oficial al confrontar a cada joven y su rendimiento con los niveles esperados de conocimiento. Por otro lado, los detalles del funcionamiento de las prácticas educativas debían llegar a que cada niño pudiera operar como el ser cognitivo racional que proponía el currículo. En otro escrito hemos analizado cómo la noción de espacio y espacialidad que ha sido parte del currículo de matemáticas colombiano, con su énfasis en el logro de la comprensión del espacio como una entidad abstracta internalizada cincela en la mente, el cuerpo y el ser del niño las marcas de un sujeto cosmopolita, sin hogar, que puede verse a sí mismo como un punto en relación con un espacio vacío de práctica social o cultural, pero si geometrizado y lleno de leyes Euclideanas (VALERO et al., 2012). Así, el currículo, con una 
ontología estructuralista sistémica de las matemáticas para organizar los contenidos escolares, y una epistemología piagetiana como teoría sobre el desarrollo del pensamiento (matemático) del niño, podría organizar la fabricación del niño racional, cognitivo moderno. Con este intento también se marcó la entrada fuerte de la racionalidad de las ciencias educativas modernas a Colombia y en particular en la educación matemática.

Desde los 1980, el currículo oficial colombiano en matemáticas ha incorporado nociones que navegan en las discusiones de la investigación internacional en educación matemática. Las epistemologías sobre las cuales construye el currículo siguen arraigadas en las teorías constructivistas del aprendizaje derivadas de la epistemología ontogenética piagetiana. Durante los 1990, las reformas curriculares adicionaron el lenguaje de los logros mínimos como parte de la agenda internacional de la Educación para todos de la UNESCO (VALERO, 2012). En la última década, el lenguaje de las competencias y los estándares que hace parte de la lógica de la Organización de Cooperación para el Desarrollo Económico (OCDE), se han infiltrado en los documentos de política educativa y en los currículos oficiales como parte de una alineación de las políticas nacionales educativos a los nuevos requisitos económicos de la sociedad global competitiva (OECD, 1989). En todas estas aparentes modificaciones la continuidad que sigue tomando nuevas formas es el supuesto de que la educación en general y en especial la educación matemática son centrales tanto para el progreso del individuo, como para la mejora de la sociedad. En todas estas formulaciones sigue estando presente la esperanza de que en las aulas de matemáticas se fabrique al niño racional, efectivo, global, incluso emprendedor, cosmopolita del siglo XXI.

\section{In(ex)clusión, subjetividad y matemáticas escolares}

En este momento surge la pregunta de cuál es la contribución de este tipo de estudios y por qué parece el análisis haber cierto tono de crítica sobre el efecto del currículo de matemáticas. Después de todo, ¿no es acaso preferible y de hecho deseable tener individuos que razonen y sean útiles a la sociedad? ¿Es acaso la intención abogar por el ser irracional, ilógico, guiado por la creencia y no capaz de ver ni esperar un futuro? ¿No es acaso laudable constatar que la educación matemática de hecho sí cumple esa función en la configuración social del momento?

Aquí, necesitamos volver a algunas de las ideas sobre el poder en Foucault (2000). Constatar que, de hecho, el currículo escolar a través de las matemáticas escolares sí insertan 
a los niños y niñas en una cierta racionalidad permite afirmar que como prácticas de gobierno, el currículo genera efectos positivos en tanto construye y crea subjetividades. El poder no es sólo destructivo u opresor, es también positivo y creativo (FOUCAULT; FAUBION, 2000). La otra cara de la moneda es, no obstante, también un resultado mismo de poder: todo sistema de razón que fabrica una narrativa de redención y formula una tesis sobre quiénes son los sujetos deseados opera una norma. Y toda narrativa pastoral, subjetividad y norma encarnan en sí mismas su opuesto como aquello que se teme y que, por lo tanto debe evitarse. Popkewitz (2008) propone la noción de abyección para referirse al efecto de exclusión que se genera a partir de la enunciación de aquello o aquellos que claramente se enmarcan dentro de la narrativa de lo deseado. Esto significa que el efecto de todo discurso que aboga por una inclusión de por sí define lo que no está incluido. Lo que no está incluido es, justamente, aquello que se desea redimir para que haga parte de lo deseado. Así, por ejemplo, cuando el discurso internacional en educación matemática afirma que las matemáticas son para todos, o los documentos curriculares se refieren a lo que debería lograr toda la población estudiantil de un país, tales enunciados efectúan de por si una categoría de exclusión de todos aquellos para quienes las matemáticas no son una posibilidad, y todos aquellos que no lograrán lo esperado (VALERO, 2013). En otras palabras, todo enunciado engendra su opuesto. Inclusión y exclusión son inseparables; de ahí el uso del término in(ex)clusión.

Esta observación es de gran importancia para mirar la educación matemática en contextos como los latinoamericanos. En primer lugar, el hecho de que las matemáticas escolares fabriquen la idea del niño racional, cosmopolita moderno implica la formación de tesis culturales sobre el tipo de yo que es deseable. Al mismo tiempo, e implícitamente, lo que estos enunciados hacen es decir quién es el yo no deseado: el niño cuyas formas de vida son diferentes y, tal vez, incluso irreconciliables con aquella forma de vida y de ser deseada. No es de extrañar, entonces, la persistente relación entre quienes son los niños que tienen éxito en las matemáticas escolares - es decir, aquellos cuya conducta, cuerpos, mentes y voluntades se conducen para conocer según las formulaciones de los currículos nacionales o de las expectativas de logro internacionales - como las de las pruebas PISA. Sistemáticamente son los niños de sectores sociales y económicos dominantes quienes alcanzan los resultados esperados, mientras que la mayoría de aquellos en posiciones diferentes, dada una multiplicidad de categorías y categorizaciones, están lejos de alcanzar los umbrales del éxito escolar deseado y establecido como norma. Las explicaciones del fracaso escolar en matemáticas de ciertos sectores de la población no es, entonces, un caso de deficiencia 
cognitiva, económica ni cultural, sino es una característica misma de la microfísica del poder en tales sociedades. En otras palabras, el fracaso escolar, y con él la exclusión, es, ante todo, una configuración misma de las normas culturales de cierta época. La exclusión es, entonces, una condición de las relaciones de poder desplegadas en la institución de la escuela moderna y de las matemáticas escolares. No es la consecuencia de atributos individuales como se ha querido argumentar.

Cuando esta formación se presenta sobre la trama histórica de las estrategias de asimilación y de negación de las diversidades anteriormente mencionadas, estar en la posición de sujeto que se aleja de las normas de razón encarnadas en el niño cosmopolita moderno es algo perfectamente serio. Las propuestas de remediar la situación con más currículo, pedagogía o didáctica de las matemáticas a la larga no hacen sino dar más de lo mismo que ya sabemos que no funciona. El punto principal no es el de, entonces, poder diseñar lo que sí puede funcionar, aunque hemos hecho algunas reflexiones al respecto (VALERO et al., 2012). El gran llamado es a abrir una posibilidad para dejar de tomar como necesario y natural la manera como hemos pensado en el empoderamiento a través de la educación matemática y en las clasificaciones y selecciones que el éxito en las matemáticas tienen. Sin una distancia que nos permita pensar que es posible dar cabida a otras subjetividades, las matemáticas escolares seguirán perpetuando exclusiones similares a las que nuestras sociedades sufren desde los mismos tiempos de la colonia. Y la promesa de una posibilidad mejor de mundo y de vida seguirá siendo el privilegio de los mismos pocos.

\section{Agradecimientos}

Agradecemos a Alexandre Pais de la Universidad Metropolitana de Manchester, Reino Unido, a Gelsa Knijnik de la Universidad del Valle do Rio dos Sinos, Brasil, y a Alex Montecino y Melissa Andrade de la Universidad de Aalborg, Dinamarca, por sus comentarios y contribuciones a las ideas presentadas en este texto. Este texto ha sido producido como parte del proyecto Estudio del papel de los escenarios y ambientes de aprendizaje de las matemáticas en los procesos de inclusion financiado por el Instituto Colombiano para el Avance de la Ciencia y la Tecnología y la Universidad de Aalborg, Dinamarca. 


\section{Referencias}

AHERN, E. El desarrollo de la educación en Colombia: 1820-1850. Revista Colombiana de Educación, Bogotá, v. 22-23, p. 7-28, 1991.

BISHOP, A. J. Las matemáticas occidentales: el arma secreta del imperialismo cultural. In: BISHOP, A. J. Aproximación sociocultural a la educación matemática. Cali: Universidad del Valle, 2005. p. $27-41$.

BRASIL. Ministério da Educação. Secretaria de Educação Básica. Parâmetros curriculares nacionais - Terceiro e quarto ciclos do ensino fundamental - Matemática. Brasília: MEC/SEF, 1998.

CARRASQUILLA, R. M. Sobre el moderninsmo. Obras completas. Santafé de Bogotá: Imprenta nacional, 1916.

DASTON, L.; GALISON, P. Objectivity. New York: Zone Books, Distributed by the MIT Press, 2007. $501 \mathrm{p}$.

DELEUZE, G.; GUATTARI, F. A thousand plateaus: capitalism and schizophrenia. Minneapolis: University of Minnesota Press, 1987. xix, 610 p.

DÍAZ, A. Democracia y nación en el siglo XIX colombiano. La ciudad: Periodismo inédito, 2010.

DUARTE, C. A "realidade" nas tramas discursivas da educação matemática. São Leopoldo: Universidade do Vale do Rio dos Sinos, 2009.

FOUCAULT, M. The order of things. An archaeology of the human sciences. New York: Vintage Books, 1971. xxiv. 387 p.

FOUCAULT, M. The subject and power. Critical Inquiry, Chicago, v. 8, n. 4, p. 777-795, 1982.

FOUCAULT, M. Technologies of the self. En: FOUCAULT, M.; RABINOW, P. (Ed.). Ethics: Subjectivity and truth. New York: The New Press, 1997. p. 223-251.

FOUCAUlT, M.; FAUBION, J. D. Power. New York: New Press, 2000. 484 p.

GARCÍA, G. Currículo y evaluación en matemáticas. Un estudio en tres décadas en la educación básica. Bogotá: Cooperativa Editorial Magisterio, 2003.

GÓMEZ, P. Los organizadores del currículo en matemáticas. Revista EMA, Santafé de Bogotá, v. 5, n. 3, p. 267-277, 2000.

GUERRA, C. Hacia una sociología del sujeto: Democracia y sociedad civil. En: LEÓN, E.;

ZEMELMAN, H. (Ed.). Subjetividad: Umbrales del pensamiento social. Barcelona: Anthropos, 1997. p. 107-136.

GUILLÉN, F. El poder político en Colombia. 4. ed. Bogotá: Planeta, 1996.

HARDY, T. "There's no hiding place". Foucault's notion of normalization at work in a mathematics lesson. In: WALSHAW, M. (Ed.). Mathematics education within the postmodern. Greenwich (USA): Information Age, 2004. p.103-119. 
HARRISON, P. Linneaus as a second Adam? Taxonomy and the religious vocation. Zygon, Chicago, v. 44, n. 4, p. 879-893, nov. 2009.

HOWSON, G. Mathematics: The Fight for Recognition. Mathematics in School, London, v. 3, n. 6, p. 7-9, jun. 1974.

KNIJNIK, G.; WANDERER, F.; DUARTE, C. Das invenções pedagógicas: a importância do uso de materiais concretos na educação matemática. San Leopoldo: Universidade do Vale do Rio dos Sinos, 2008.

KNIJNIK, G.; WANDERER, F.; GIONGO, C.; DUARTE, C. Etnomatemática em movimento. Belo Horizonte: Autêntica, 2012.

LAISANT, C. A.; FEHR, H. Préface. L' Enseignement Mathématique, Paris, v. 1, n. 1, p. 1-5, 1899.

MEN (MINISTERIO DE EDUCACIÓN NACIONAL DE COLOMBIA). Estándares básicos de competencias en matemáticas. Potenciar el pensamiento matemático: !un reto escolar! In: MEN (MINISTERIO DE EDUCACIÓN NACIONAL DE COLOMBIA). Estándares básicos de competencias en lenguaje, matemáticas, ciencias y ciudadanas. Guía sobre lo que los estudiantes deben saber y saber hacer con lo que aprenden. Bogotá: Ministerio de Educación Nacional de Colombia, 2006. p. 46-95.

MOLANO, M. Carlos Eduardo Vasco Uribe. Trayectoria biográfica de un intelectual colombiano: Una mirada a las reformas curriculares en el país. Revista Colombiana de Educación, Bogota, v. 61, p. 161-198, 2011.

MUTIS, J. C.; FONNEGRA, G. Mutis y la expedición botánica: Documentos. Bogotá: El Áncora Editores, 1983.

NIETO, M. Remedios para el Imperio. De las creencias locales al conocimiento ilustrado en la botánica del siglo XVIII. In: OBREGÓN, D. (Ed.). Culturas científicas y saberes locales. Bogotá: Universidad Nacional de Colombia - Programa Universitario de Investigación en Ciencia, Tecnología y Cultura, 2000. p. 89-102.

OECD. Education and the economy in a changing society. Paris: OECD, 1989.

PAIS, A.; VALERO, P. Researching research: mathematics education in the Political. Educational Studies in Mathematics, Dordrecht, v. 80, n. 1, p. 9-24, Mar. 2012.

POOVEY, M. A history of the modern fact: problems of knowledge in the sciences of wealth and society. Chicago: University of Chicago Press, 1998, 419 p.

POPKEWITZ, T. S. The Alchemy of the Mathematics Curriculum: Inscriptions and the Fabrication of the child. American Educational Research Journal, Washington, v. 41, n. 1, p. 3-34, Mar. 2004.

POPKEWITZ, T. S. Cosmopolitanism and the age of school reform: science, education, and making society by making the child. New York: Routledge, 2008, $220 \mathrm{p}$.

RADFORD, L. From truth to efficiency: Comments on some aspects of the development of mathematics education. Canadian Journal of Science, Mathematics and Technology Education, Toronto, v. 4, n. 4, p. 551-556, Jan. 2004. 
RADFORD, L. Culture and cognition: Towards and anthropology of mathematical thinking. In: ENGLISH, L. D.; BARTOLINI BUSSI, M. G. (Ed.). Handbook of international research in mathematics education. 2. ed. New York, NY: Routledge, 2008a. p. 439-464.

RADFORD, L. The ethics of being and knowing: Towards a cultural theory of learning. In: RADFORD, L.; SCHUBRING, G. (Ed.). Semiotics in Mathematics Education: Epistemology, History, Classroom, and Culture. Rotterdam: Sense, 2008b. p. 215-234.

RIVAS, J. Pedagogía de la dignidad de estar siendo. Entrevista con Hugo Zemelman y Estela Quintar. Revista interamericana de educación de adultos, Patzcuaro, Mexico, v. 27, n. 1, p. 113-140, ene./jun. 2005.

RÜEGG, W. (Ed.). A history of the university in Europe. Voleume III: Universities in the nineteenth and early twentieth centiries (1800-1945). 3 ed. Cambridge: Cambridge University Press, 2004.

SAAR, M. Genealogy and subjectivty. European Journal of Philosophy, Oxford, v. 10, n. 2, p. 231245, Dec. 2002.

SALDARRIAGA-VÉLEZ, Ó. Subjetividad/objetividad. Hipótesis para una lectura del "campo epistémico" en Colombia-siglo XIX. In: SÁNCHEZ LOPERA, A.; HENSEL RIVEROS, F. D.; (Ed.). Actualidad del sujeto. Conceptualizaciones, genealogías y prácticas. Bogotá: Editorial Universidad de Rosario, 2010. p. 79-118.

SÁNCHEZ, C. H.; ALBIS, V. Historia de la enseñanza de las matemáticas en Colombia. De Mutis al siglo XXI. Quipu, Mexico D.F., v. 14, n. 1, p. 109-157, ene./abr. 2012.

SKOVSMOSE, O. Hacia una filosofía de la educación matemática crítica. Bogotá: una empresa docente, 1999, $253 \mathrm{p}$.

SKOVSMOSE, O.; VALERO, P. Rompimiento de la neutralidad política: el compromiso crítico de la educación matemática con la democracia. In: VALERO, P.; SKOVSMOSE, O. (Ed.). Educación matemática crítica: Una visión socio-política del aprendizaje y enseñanza de las matemáticas. Bogotá: Ediciones Uniandes, 2012. p. 25-61.

SLOAN, P. R. The Buffon-Linnaeus Controversy. Isis, Chicago, v. 67, n. 3, p. 356-375, Sept. 1976.

VALERO, P. Discourses of power in mathematics education research: Concepts and possibilities for action. PNA. Revista de investigación en didáctica de la matemática, Granada, España, v. 2, n. 2, p. 43-60, Jan. 2008.

VALERO, P. En medio de lo global y lo local: las políticas de la reforma en la educación matemática en una sociedad globalizada. In: VALERO, P.; SKOVSMOSE, O. (Ed.). Educación matemática crítica: Una visión socio-política del aprendizaje y enseñanza de las matemáticas. Bogotá: Ediciones Uniandes, 2012. p. 83-105.

VALERO, P. Mathematics for all and the promise of a bright future. In: CONGRESS OF EUROPEAN RESEARCH IN MATHEMATICS EDUCATION - CERME, 8., 2013, Antalya, Turkey. Proceedings of the: 2013. Antalya, Turkey: UBUZ, B.; HASER, Ç.; MARIOTTI, M. (Ed.), 2013, p. 1804-1813. Disponible en: <http://www.mathematik.unidortmund.de/ erme/doc/CERME8/CERME8_2013_Proceedings.pdf $>$. Consultado el: 20 jul. 2014. 
VALERO, P.; GARCIA, G.; CAMELO, F.; MANCERA,G.; ROMERO, J. Mathematics education and the dignity of being. Pythagoras. Journal of the Association for Mathematics Education of South Africa, Johannesurg, v. 33, n. 2, p. 1-9, Nov. 2012.

WALSHAW, M. Pre-service Mathematics Teaching in the Context of Schools: An Exploration into the Constitution of Identity. Journal of Mathematics Teacher Education, Netherlands, v. 7, n. 1, p. 63-86, Mar. 2004. 